\title{
IMPROVEMENT OF CRACK RESISTANCE OF BANDED SUPPORTING ROLLS AT HIGH-SPEED SURFACING WITH LOW HEAT INPUT
}

\author{
S.V. SHCHETININ \\ Priazovsky State Technical University \\ 7 Universitetskaya Str., 87500, Mariupol, Ukraine. E-mail: schetininSergey2012@yandex.ua
}

\begin{abstract}
Improvement of crack resistance of banded supporting rolls is an urgent problem. The work is a study of the mechanism of improvement of deposited metal crack resistance with the aim of development of the process of high-speed surfacing of supporting rolls with low heat input. The method of X-ray structural analysis in diffractometer DRON-3 revealed that the electrode shape and heat input at surfacing have a considerable influence on microdistortions of crystalline lattice, which are responsible for microstresses. The latter lead to intensive formation and propagation of cracks, their initiation mechanism being associated with dislocations. Established regularities were confirmed at measurement of dislocation density by broadening of X-ray lines. Minimum crystalline lattice microdistortions, microstresses and dislocation density are achieved in surfacing with wire and composite electrode at a high speed and with minimum heat input. At increase of surfacing speed and lowering of heat input the rate of heating, cooling and solidification of liquid metal in the weld pool becomes higher, that provides microstructure refinement and increase of deposited metal crack resistance. A process of high-speed surfacing of banded supporting rolls with low heat input was proposed, providing an increase of crack resistance and preventing band failure. 13 Ref., 1 Table, 6 Figures.
\end{abstract}

Keywords: high-speed surfacing with low heat input, crystalline lattice microdistortions, microstresses, dislocation density, welding stresses, crack resistance, banded supporting rolls

Supporting rolls, which prevent sagging and breaking of working rolls, operate under the conditions of high specific stresses, therefore, surfacing of supporting rolls was not performed, particularly, of banded supporting rolls, which are manufactured by placing the band on the axle with interference fit. This results in stresses developing in the band, which may lead to failure. Therefore, improvement of crack resistance of banded supporting rolls is an important scientific-technical problem.

High-speed surfacing with a low heat input is an effective technique to improve crack resistance. However, its impact on deposited metal structure and properties has not been studied well enough [1-8].

The aim of this research is studying the mechanism of improvement of deposited metal crack resistance and development of the process of high-speed surfacing of banded supporting rolls with low heat input.

Electrode shape is one of surfacing process parameters, as the arc moving over the electrode tip, is concentrated in surfacing with wire, and in deconcentrated in surfacing ribbon electrode, depending on the position of which the arc moves along the longitudinal axis, or across the weld pool. Arc movement determines the heat input into the pool, and in constant surfacing mode also the rate of heating and cooling of liquid metal and HAZ. This changes the pool solidification conditions, diffusion processes, structural and phase transformations $[5,6]$, and deposited metal quality. However, the influence of electrode shape on deposited metal quality has not been sufficiently studied [7,8].

(C) S.V. SHCHETININ, 2016
Moreover, it is common knowledge that at heat input lowering the probability of cold cracking becomes higher, as a result of cooling rate increase.

However, Prokhorov [5] notes that a considerable lowering of heat input in welding and the respective increase of cooling rate may lead to lowering of cold cracking probability.

Electrode shape and heat input were found to have a considerable influence on arc movement, thermal cycles and solidification rate, which becomes higher with increase of welding speed as follows: $v_{\text {sol }}=\cos \Delta v_{\mathrm{w}}$ (Figure 1). At surfacing with the same heat input by a perpendicular ribbon electrode, the rates of heating, cooling, and solidification are maximum. At surfacing by a longitudinal ribbon electrode, the arc movement along the weld pool results in increase of heat input into the side edges of the pool, and the rate of metal heating, cooling and solidification decreases. At surfacing with wire electrode, the rate of heating, cooling and solidification decreases even more, as a result of concentration and increase of heat input effectiveness. Minimum rate of heating, cooling and solidification is achieved in surfacing with a composite electrode, as a result of arc movement along rectilinear sections of ribbon electrode in the area of pool side edges. At a constant heat input, cooling rate decreases from $300 \mathrm{deg} / \mathrm{s}$ at surfacing with a perpendicular ribbon electrode to $60 \mathrm{deg} / \mathrm{s}$ at surfacing by a composite electrode.

With increase of welding speed and lowering of heat input, the rates of heating and cooling rise (Figure 2). Here, the heating rate rises much more - from 157 up to $500 \mathrm{deg} / \mathrm{s}$. Cooling rate rises from $53 \mathrm{up}$ to $120 \mathrm{deg} / \mathrm{s}$. 


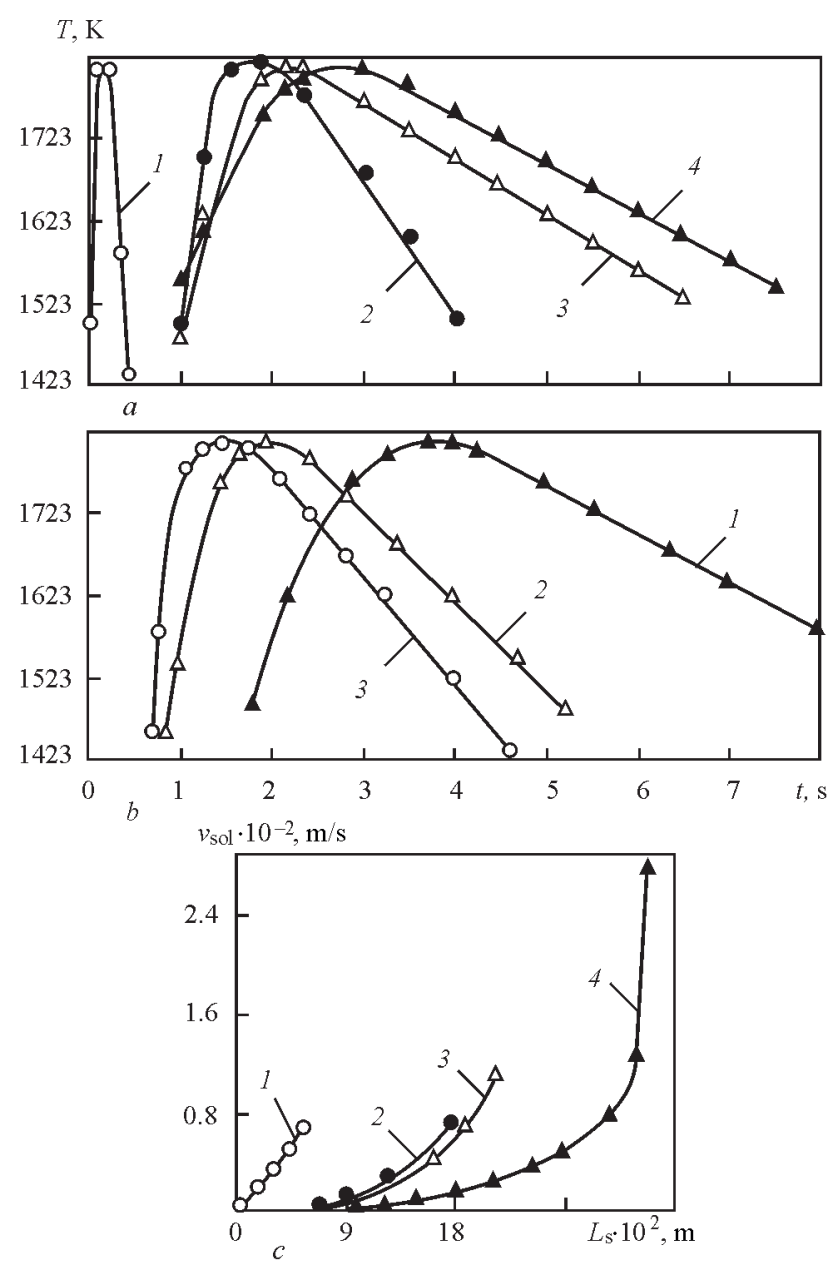

Figure 1. Regularity of impact of electrode shape on thermal cycle $(a)$ and solidification rate of weld pool liquid metal (c) (1 - perpendicular ribbon electrode; 2 - longitudinal ribbon electrode; 3 - wire; 4 - composite electrode), and of heat input $(1-3.6 ; 2-2.7 ; 3-1.8 \mathrm{MJ} / \mathrm{m})$ on thermal cycle with composite electrode $(b)$

At high-speed surfacing the microstructure of deposited metal and HAZ is refined, that is the consequence of increase of heating and cooling rate, and greater number of solidification centers, which, being located ahead of the front of growing columnar crystallites, inhibit their further growth. At heating pearlite and ferrite transform into austenite. As a result of greater heating rate, austenite grains do not have enough time to grow, and austenite stability decreases. At cooling decomposition of unstable fine-grained austenite proceeds in the upper subcritical temperature range with formation of sorbite and pearlite, that prevents cracking.

At increase of welding speed and lowering of heat input, increase of heating and cooling rate results in increase of solidification rate (Figure 3), microstructure refinement and increase of deposited metal crack resistance.

Refinement of microstructure at high-speed surfacing with low heat input is accompanied by simultaneous lowering of welding stresses, the nature of formation of which is not fully understood.

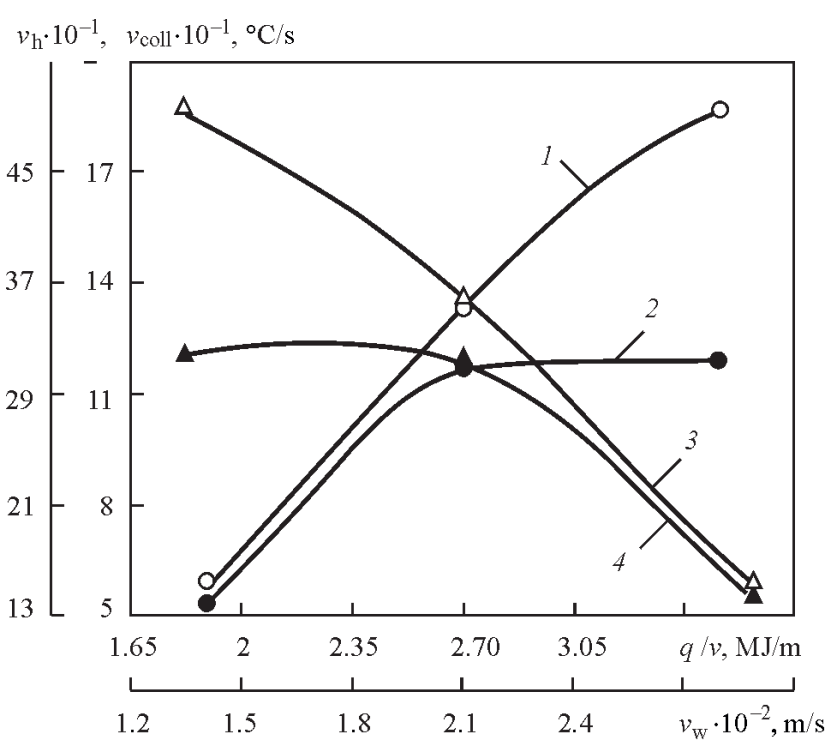

Figure 2. Dependence of the rate of heating $(1,3)$ and cooling $(2$, $4)$ of metal on welding speed $(1,2)$ and heat input $(3,4)$

As a result of heat input during surfacing the metal is exposed to thermodeformational cycle, which is responsible for crystalline lattice microdistortions and microstresses. Thermodeformational cycle leads to development of inherent deformations and microstresses in the deposited metal, which determine crystalline lattice microdistortions [9]:

$$
\sigma=E \frac{\Delta a}{a}, \mathrm{MPa},
$$

where $E=19.68 \cdot 10^{4} \mathrm{MPa}$ is the modulus of elasticity; $\Delta a / a$ are the crystalline lattice microdistortions.

Crystalline lattice microdistortions were determined at five-layer surfacing on plates of $(30 \times 300 \times 400) \cdot 10^{-3} \mathrm{~m}$ size with Sv08A wire of $4 \cdot 10^{-3} \mathrm{~m}$ diameter, with $08 \mathrm{kp}$ ribbon electrode of $(0.5 \times 45) \cdot 10^{-3}$ $\mathrm{m}$ size, positioned in the longitudinal or perpendicular directions, and composite electrode. Chemical composition of material used is given in the Table. Surfacing was performed using ceramic flux $\mathrm{ZhSN}-$

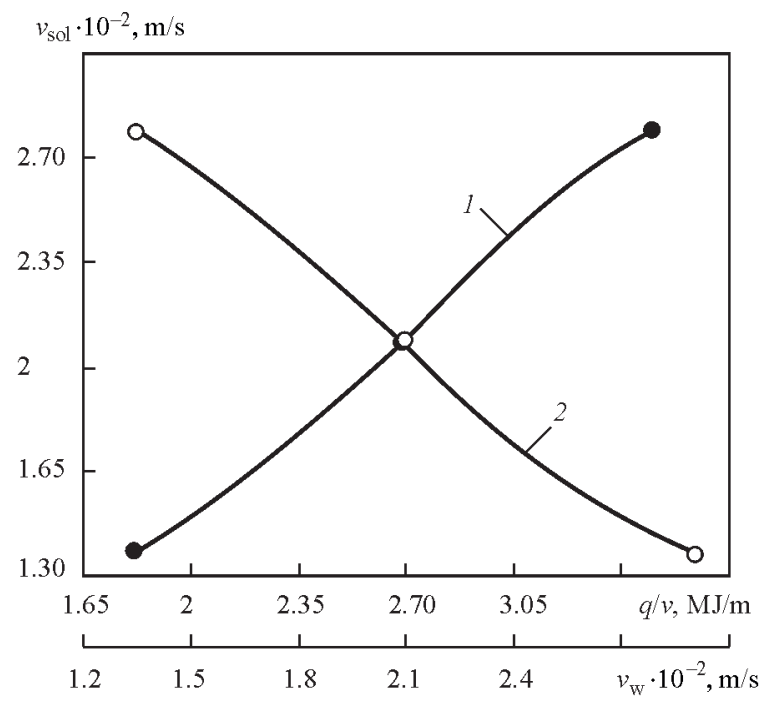

Figure 3. Dependence of solidification rate of weld pool liquid metal on welding speed (1) and heat input (2) 
Composition of surfacing consumables, wt.\%, acc. to GOST 2246-80, GOST 503-81, GOST 1050-88

\begin{tabular}{|c|c|c|c|c|c|c|}
\hline Material & $\mathrm{C}$ & $\mathrm{Mn}$ & $\mathrm{Si}$ & $\mathrm{Cr}$ & $\mathrm{S}$ & $\mathrm{P}$ \\
\hline Sv08A wire & $\leq 0.1$ & $0.35-0.60$ & $\leq 0.03$ & $\leq 0.12$ & \multirow{2}{*}{0.025} & \multirow{2}{*}{0.030} \\
\hline 08kp ribbon electrode & $0.05-0.12$ & $0.25-0.50$ & $\leq 0.03$ & $\leq 0.10$ & & \\
\hline
\end{tabular}

5 in optimum modes characteristic for each process: for wire electrode - at $I=650-750 \mathrm{~A}, U_{\mathrm{a}}=31-33 \mathrm{~V}$ and $v_{\mathrm{s}}=(0.56,0.83,1.1) \cdot 10^{-2} \mathrm{~m} / \mathrm{s}$; for longitudinal and perpendicular ribbon electrode $-I=450-550 \mathrm{~A}$, $U_{\mathrm{a}}=29-31 \mathrm{~V}$ and $v_{\mathrm{s}}=(0.33,0.5,0.67) \cdot 10^{-2} \mathrm{~m} / \mathrm{s}$; for composite electrode $-I=1950-2050 \mathrm{~A}, U_{\mathrm{a}}=29$ $31 \mathrm{~V}$ and $v_{\mathrm{s}}=(1.4,2.1,2.8) \cdot 10^{-2} \mathrm{~m} / \mathrm{s}$. Heat input for each surfacing process changed within $q / v=1.8,2.7$ and $3.6 \mathrm{MJ} / \mathrm{m}$.

In order to control deposited metal quality, investigations of the influence of electrode shape and heat input on structural and phase transformations were conducted by measurement of crystalline lattice microdistortions, dislocation density, microstructure, microhardness of deposited metal and HAZ. Investigations of the value of crystalline lattice microdistortions were performed by the method of X-ray structural analysis in diffractometer DRON-3.

In arc surfacing vacancies form in the deposited metal under the impact of thermal excitation [5], as the energy of activation of vacancy generation is smaller than that of formation of interstitial atoms. In the zone of vacancy generation, static equilibrium of interatomic interaction forces is disturbed, that leads to displacement of adjacent atoms from their equilibrium positions and crystalline lattice microdistortions.

As was established, electrode shape and heat input have a significant influence on crystalline lattice microdistortions (Figure 4,a). At surfacing with perpendicular ribbon electrode at a low speed, high heat input results in metal oversaturation with excess vacancies [5], and crystalline lattice microdistortions are maximum. At surfacing with a longitudinal ribbon electrode, low- ering of heat input leads to reduction of crystalline lattice microdistortions. Crystalline lattice microdistortions are even smaller at surfacing with wire at a higher speed. Minimum microdistortions of the crystalline lattice are achieved in surfacing with a composite electrode at a high speed and minimum heat input.

Change of heat input at surfacing with ribbon electrode, positioned perpendicular to and along the surfacing direction, does not influence crystalline lattice microdistortions, as a result of metal oversaturation with vacancies. Increase of the speed of surfacing with wire and composite electrode influences crystalline lattice microdistortions, which decrease with increase of surfacing speed and lowering of heat input. Minimum microdistortions of crystalline lattice are provided in surfacing with a composite electrode at speed of $2.1 \cdot 10^{-2} \mathrm{~m} / \mathrm{s}$, due to lowering of heat input and generation of Schottky vacancies.

In keeping with the influence of electrode shape and heat input on crystalline lattice microdistortions, maximum microstresses arise in the deposited metal in surfacing with a perpendicular ribbon electrode (Figure $4, b$ ). In surfacing with a longitudinal ribbon electrode microstresses become smaller. In surfacing with wire electrode microstresses become even smaller. Microstresses decrease the most significantly in surfacing with a composite electrode with low heat inputs, as the level of plastic deformation decreases at lowering of heat input and accelerated cooling [10], that prevents failure.

Microstresses lead to intensive formation and propagation of cracks $[5,6]$, the mechanism of initiation of which is associated with dislocations [11].
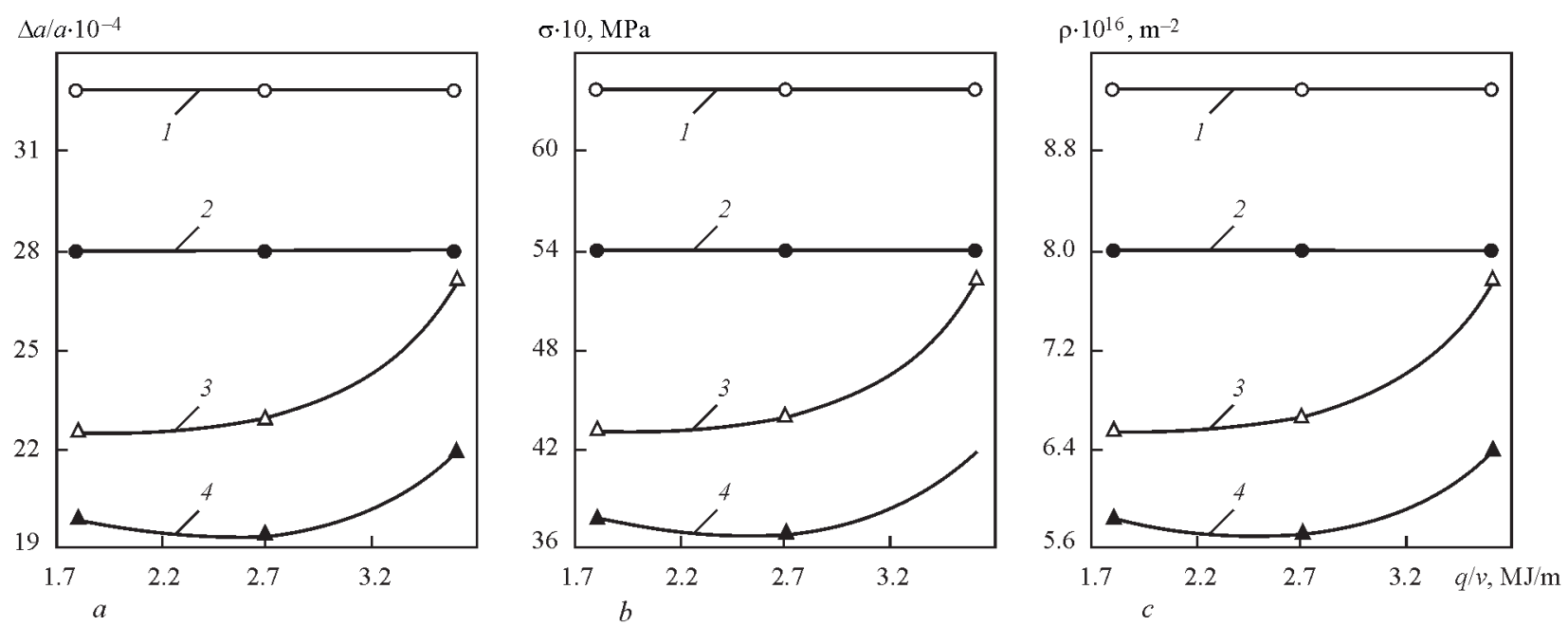

Figure 4. Regularities of impact of electrode shape and heat input on crystalline lattice microdistortions $(a)$, microstresses $(b)$ and dislocation density $(c)$ in deposited metal: 1 - perpendicular ribbon electrode; 2 - longitudinal ribbon electrode; 3 - wire; 4 composite electrode 
Studying the influence of electrode shape and heat input on dislocation density was performed by broadening of X-ray lines [12]:

$$
\rho=3.46 \cdot 10^{19} \beta_{211}^{2}, \mathrm{rad} \cdot \mathrm{m}^{-2} .
$$

As a result it was established that maximum dislocation density is characteristic for surfacing with a perpendicular ribbon electrode, and decreases in surfacing with a longitudinal ribbon electrode (Figure 4, c). Dislocation density decreases in surfacing at wire and, particularly, with composite electrode with low heat input. This is in keeping with the influence of electrode shape and heat input on crystalline lattice microdistortions, as the totality of vacancies and nonmetallic inclusions (NMI) is the source of dislocations.

Assessment of the degree of deposited metal contamination by NMI and determination of their chemical composition were performed to clarify the established change of dislocation density.

Evaluation of the degree of deposited metal contamination by NMI was performed in optical microscope «Vertivol» at $\times 500$ magnification by a linear method. Contamination index was calculated as a ratio of total length of inclusions to total counted length, and chemical composition of NMI was determined by the method of X-ray spectral analysis in SEM-100U microscope and by the method of laser microspectral analysis in laser microspectroanalyzer LMA-10.

Examination in optical microscope at $\times 400, \times 500$ magnification and in SEM at $\times 1000$ magnification revealed that the deposited metal has NMI, which can be subdivided into two kinds: single spherical luminescent of more than $3 \mu \mathrm{m}$ size (Figure 5,a) and a large number of spherical nontransparent ones of less than $1 \mu \mathrm{m}$ size (Figure 5, $b$ ).

Degree of deposited metal contamination decreases with increase of surfacing speed, as a result of intensive convective flows and speed of liquid metal motion. The main NMI are manganese and silicon. Established influence of electrode shape and heat input on the degree of deposited metal contamination by NMI is in good agreement with their impact on dislocation density.

Established regularity of the impact of electrode shape and heat input on dislocation density leads to the conclusion that electrode shape and heat input affect deposited metal crack resistance, as a result of their influence on arc movement, thermal cycles and solidification rate.

It was found that microstructure dispersion (Figure 6) and deposited metal crack resistance are increased at increase of heating and cooling rate.

At surfacing with a longitudinal ribbon electrode, deposited metal microstructure is coarse-grained and non-uniform (Figure 6, a). In surfacing by a perpendicular ribbon electrode the microstructure is slightly refined, while remaining coarse-grained (Figure 6,b). Surfacing with wire and composite electrode leads to refinement of

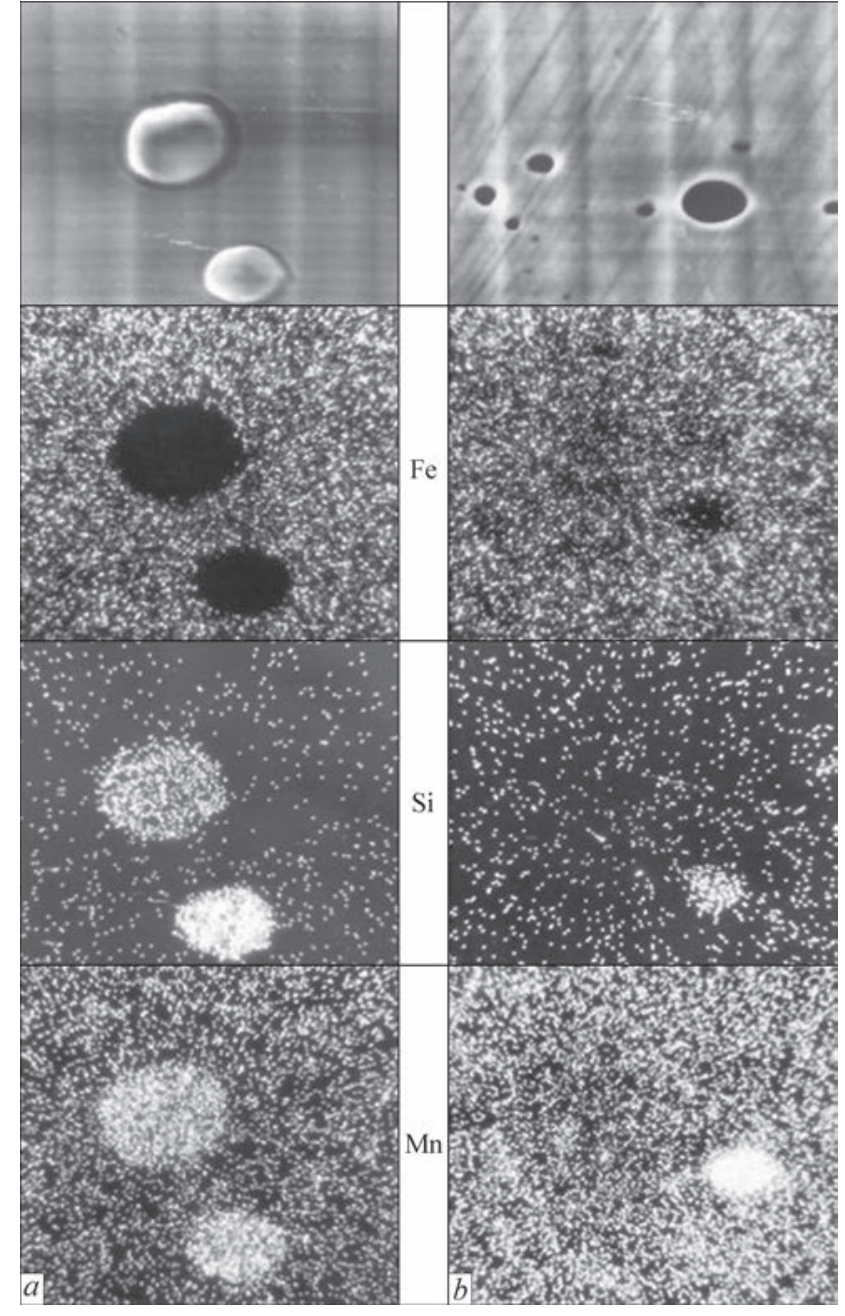

Figure 5. Nonmetallic inclusions of the size of more than $3 \mu \mathrm{m}$ $(a-\times 1500$ with $1 / 2$ red $)$ and less than $1 \mu \mathrm{m}(b-\times 4000$ with $1 / 2$ red) in X-ray spectrograms of distribution

microstructure (Figure 6, $c, d$ ), which is a ferrite-cementite mixture and is fine-grained and uniform.

Microstructure of deposited metal and HAZ is refined in a similar way in high-speed surfacing, that is the consequence of higher heating and cooling rate and greater number of solidification centers, which, being located ahead of the front of growing columnar crystallites, inhibit their further growth.

As a result of higher heating rate, austenite grains do not have enough time to grow, and austenite stability decreases. At cooling, decomposition of unstable fine-grained austenite occurs in the upper subcritical temperature range with formation of sorbite and pearlite, that prevents cracking. Minimum crystalline lattice microdistortions, microstresses, dislocation density and fine-grained uniform structure, result in increase of crack resistance.

In electric arc surfacing of banded supporting rolls of mill 3000 with higher heat input the band broke up.

To increase crack resistance and wear resistance of banded supporting rolls, an energy-saving technique of high-speed surfacing with a low heat input was developed [13]. High-speed surfacing of band- 


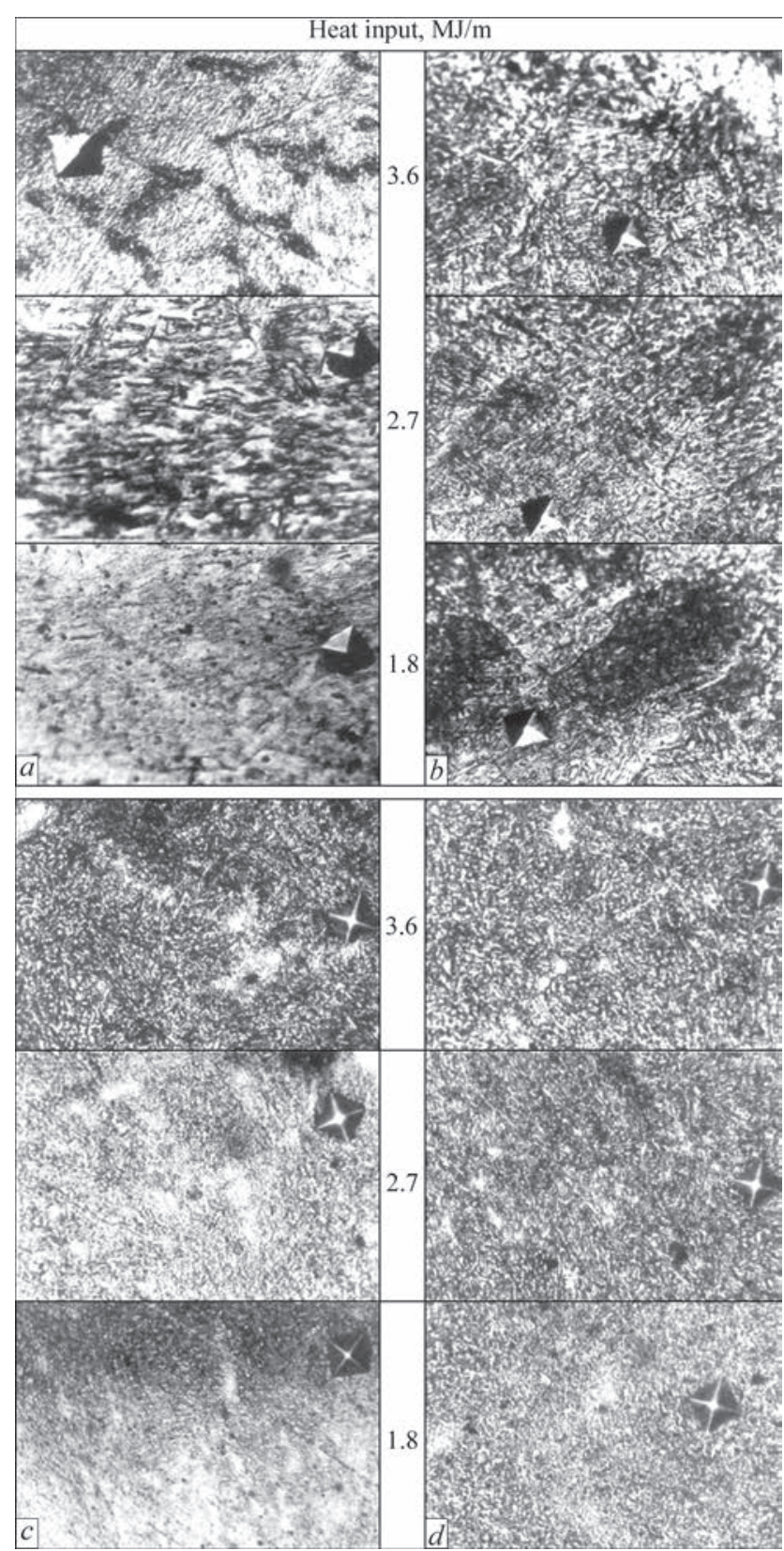

Figure 6. Microstructure $(\times 300)$ of metal deposited with longitudinal $(a)$, perpendicular $(b)$ ribbon electrode, wire $(c)$ and composite electrode $(d)$ with different heat input

ed supporting rolls of mill 3000, which consist of an axle and band from steel $90 \mathrm{KhF}$, was performed with preheating and concurrent heating up to $300-350^{\circ} \mathrm{C}$. First a buffer layer was deposited with low-carbon wire Sv-08G2S of $5 \mathrm{~mm}$ diameter using AN-60 flux, followed by a wear-resistant layer, deposited with flux-cored wire PP-Np-25Kh5FMS of $3.6 \mathrm{~mm}$ diameter using AN-26P flux with heat input of $1.1 \mathrm{MJ} / \mathrm{m}$ at $I=750-800 \mathrm{~A}, U_{\mathrm{a}}=30-32 \mathrm{~V}, v_{\mathrm{s}}=75 \mathrm{~m} / \mathrm{h}$. Surfacing was followed by heat treatment and delayed cooling. In high-speed surfacing of banded supporting rolls with low heat input the band did not break, that confirms the effectiveness of the process.
Developed energy-saving process of high-speed surfacing of banded supporting rolls with low heat input ensures minimum crystalline lattice microdistortions, microstresses, dislocation density and welding stresses, fine-grained uniform structure, high crack resistance and prevention of band failures.

\section{Conclusions}

1. Mechanism of improvement of deposited metal crack resistance in high-speed surfacing with low heat input due to lowering crystalline lattice microdistortions, microstresses, dislocation density and welding stresses was established. Minimum crystalline lattice microdistortions, microstresses and dislocation density are achieved in high-speed surfacing with low heat input with wire and composite electrode.

2. At increase of surfacing speed and lowering of heat input, the rate of heating, cooling and solidification of weld pool liquid metal decreases, that ensures refinement of the microstructure and higher of crack resistance.

3. A process of high-speed surfacing of banded supporting rolls with low heat input was developed, which provides lowering of welding stresses, refinement of microstructure, increase crack resistance and absence of band failures.

1. Frumin, I.I. (1961) Automatic electric arc surfacing. Moscow: Metallurgiya.

2. Frumin, I.I., Reznitsky, A.M. (1975) Application of dispersion-hardened steel for surfacing of primary mill rollers. Avtomatich. Svarka, 9, 55-57.

3. Ryabtsev, I.A. (2004) Surfacing of machine parts and mechanisms. Kiev: Ekotekhnologiya.

4. Tylkin, M.A. (1975) Improvement of life of metallurgical equipment parts. Moscow: Metallurgiya.

5. Prokhorov, N.N. (1976) Physical processes in metals during welding. Moscow: Metallurgiya.

6. Shorshorov, M.Kh., Belov, V.V. (1972) Phase transformations and changes of steel properties in welding. Moscow: Nauka.

7. Savitsky, A.M., Savitsky, M.M., Novikova, D.P. (2004) Influence of welding speed and duration of periodical cooling on structure formation in welded joints of hardening steels in arc welding with thermal cycling. The Paton Welding J., 8, 39-43.

8. Musiyachenko, V.F., Mikhoduj, L.I., Pozdnyakov, V.D. (1990) Resistance to cold cracking of T-joints of high-strength steel in shielded-gas single-pass welding. Svarochn. Proizvodstvo, 2, 13-15.

9. Gorelik, S.S., Rastorguev, L.N., Skakov, Yu.A. (1970) X-ray structural and electrooptical analysis. Moscow: Metallurgiya.

10. Myalin, M.I., Sagalevich, V.M., Turygin, V.N. et al. (1988) Influence of thermodeformation cycle on technological strength in surfacing of gray cast iron with bronze. Svarochn. Proizvodstvo, 3, 10-11.

11. Finkel, V.M. (1970) Physics of fracture. Moscow: Metallurgiya.

12. Ivanov, A.N., Mezhenny, Yu.O., Ostrov, A.E. (1987) Comparative determination of dislocations density in semicrystals by width of X-ray lines and electron microscopic analysis. Zavod. Laboratoriya, 2, 43-48.

13. Bojko, V.S., Shchetynin, S.V., Klimanchuk, V.V. Method of restoration and strengthening of cylindrical parts. Pat. 65092 Ukraine. Int. Cl. B 23 K 9/04. Publ. 15.03.2004.

Received 26.10.2016 